П. В. Ролдугин, А. В. Тарасов, О числе биюнктивных функций, инвариантных относительно данной подстановки, Дискрет. матем., 2002, том 14, выпуск 3, 23-41

DOI: https://doi.org/10.4213/dm251

Использование Общероссийского математического портала Math-Net.Ru подразумевает, что вы прочитали и согласны с пользовательским соглашением http://www . mathnet.ru/rus/agreement

Параметры загрузки:

IP : 54.172 .240 .79

26 апреля 2023 г., 13:04:32 
УдК 519.7

\title{
О числе биюнктивных функций, инвариантных относительно данной подстановки
}

\author{
() 2002 г. П. В. Ролдугин, А. В. Тарасов
}

\begin{abstract}
Класс булевых биюнктивных функций является одним из классов Шеффера. Основным качеством, делающим актуальным изучение биюнктивных функций, является следующее: задача проверки совместности системы уравнений, составленной над классом Шеффера, является полиномиальной (см., например, [1-4]). В данной работе оценивается число биюнктивных функций, содержащих в своей группе инерции относительно симметрической группы заданную подстановку. В частности, описаны свойства и подсчитано точное число биюнктивных функций, инвариантных относительно полноцикловой перестановки переменных.
\end{abstract}

\section{1. Основные понятия}

Биюнктивной функцией называется любая булева функция $f\left(x_{0}, \ldots, x_{n-1}\right)$, которая реализуется 2-КНФ, то есть может быть задана в виде

$$
f\left(x_{0}, \ldots, x_{n-1}\right)=\left(x_{i_{1}}^{\alpha_{1}} \vee x_{j_{1}}^{\beta_{1}}\right)\left(x_{i_{2}}^{\alpha_{2}} \vee x_{j_{2}}^{\beta_{2}}\right) \ldots\left(x_{i_{t}}^{\alpha_{t}} \vee x_{j_{1}}^{\beta_{t}}\right),
$$

где $t$ - некоторое натуральное число (возможно, различное для разных функщий $f$ ), $\alpha_{i}, \beta_{i} \in\{0,1\}, i=1, \ldots, t, x^{0}=\bar{x}$ и $x^{1}=x$. Определим понятие графа 2-КНФ биюнктивной функции. Данное понятие впервые было применено в [2]. Графом 2-КНФ назовем ориентированный граф $G=(V, E)$ с множеством вершин $V$ и множеством дуг $E$ такими, что

$$
\begin{aligned}
& V=\left\{x_{0}, \ldots, x_{n-1}, \bar{x}_{0}, \ldots, \bar{x}_{n-1}\right\} \\
& E=\left\{\left(x_{i_{k}}^{\alpha_{k} \oplus 1}, x_{j_{k}}^{\beta_{k}}\right), k=1, \ldots, t\right\} \cup\left\{\left(x_{i_{k}}^{\beta_{k} \oplus 1}, x_{j_{k}}^{\alpha_{k}}\right), k=1, \ldots, t\right\} .
\end{aligned}
$$

Приведенные выше определения и необходимые для данной статьи свойства этих объектов взяты из $[2,5,7]$.

Класс биюнктивных функций на данный момент изучен весьма слабо, например, неизвестны даже хорошие оценки числа биюнктивных функций от $\boldsymbol{n}$ переменных. В [5] построен критерий биюнктивности, определяющий свойства множества выполняющих векторов биюнктивной функции; в [7] изучены свойства графа 2-КНФ и рассмотрены вопросы минимизации биюнктивных функций. Кроме того, в [3] приведены все биюнктивные функции от 3-х и 4-х переменных. 
Напомним некоторые понятия теории булевых функций. Эти термины можно найти, например, в [6].

Имплицентой булевой функции $f$ называется функция $K$ такая, что $f \cdot K=f$. Имплищента $K$ называется элементарной, если она является элементарной дизъюнкцией. Элементарная имплицента $K$ называется простой имплицентой функции $f$, если для любой элементарной имплищенты $K^{\prime}$ функции $f$ такой, что $K^{\prime} \cdot K=K$, верно равенство $K^{\prime}=K$. Длиной (или рангом) элементарной имплиценты $K$ называется число существенных переменных функции $K$. Известно, что произведение всех простых имплицент совпадает с функщией $f$ и называется сокращенной КНФ функции $f$. Отметим, что в [5] было показано, что для биюнктивной функции длина любой простой имплиценты не превышает двух.

Введем следующее обозначение. Пусть дана булева функция $f$ от $n$ переменных и подстановка $s$ из группы $S_{n}$, где $S_{n}$ - симметрическая группа подстановок, действующая на множестве $\{0,1, \ldots, n-1\}$. Через $s(f)$ обозначим функцию

$$
s(f)\left(x_{0}, x_{1}, \ldots, x_{n-1}\right)=f\left(x_{s(0)}, \ldots, x_{s(n-1)}\right) .
$$

Легко видеть, что $s(f \cdot g)=s(f) s(g)$.

Группой инерции $J(f)$ булевой функщии $f\left(x_{0}, \ldots, x_{n-1}\right)$ относительно симметрической группы $S_{n}$ называется группа подстановок $s$ из группы $S_{n}$ таких, что

$$
s(f)=f .
$$

Поскольку в данной работе изучаются только указанные группы инерции, слова "относительно группы $S_{n}$ " далее будем опускать.

Если подстановка $s$ лежит в группе $J(f)$, то функцию $f$ назовем функцией, инвариантной относительно подстановки $s$.

Обозначим $D(s, n)$ число биюнктивных функщий от $n$ переменных, содержащих в своей группе инерции подстановку $s$.

Целью данной работы является вычисление или оценка числа биюнктивных функщий, инвариантных относительно фиксированной подстановки. Эту задачу можно упростить, воспользовавшись следующим утверждением.

Предложение 1. Если подстановки s и $s^{\prime}$ подобны, то $D(s, n)=D\left(s^{\prime}, n\right)$.

Доказательство. Действительно, подстановка $s$ подобна $s^{\prime}$ тогда и только тогда, когда существует подстановка $h$ из $S_{n}$ такая, что выполнено равенство $h s=s^{\prime} h$. Отметим, что равенство $s(f)=f$ выполняется тогда и только тогда, когда выполнено равенство $h s(f)=h(f)$. Аналогичную роль для подстановки $s^{\prime}$ играет равенство $s^{\prime} h(f)=h(f)$. Следовательно, подстановка $s^{\prime}$ лежит в группе $J(h(f))$. Таким образом, функщия $f$ инвариантна относительно подстановки $s$ тогда и только тогда, когда функщия $h(f)$ инвариантна относительно подстановки $s^{\prime}$. Остается заметить, что функции $f$ и $f^{\prime}$ различны тогда и только тогда, когда различны функции $h(f)$ и $h\left(f^{\prime}\right)$, поскольку $h$ - подстановка. Кроме того, функщия $f$ биюнктивна тогда и только тогда, когда функция $h(f)$ биюнктивна. Таким образом, количество биюнктивных функций, инвариантных относительно подстановки $s$, совпадает с количеством биюнктивных функций, инвариантных относительно подстановки $s^{\prime}$.

Предложение доказано.

Подстановку

$$
c_{n}=\left(\begin{array}{ccccc}
0 & 1 & \ldots & n-2 & n-1 \\
1 & 2 & \ldots & n-1 & 0
\end{array}\right)
$$


из группы $S_{n}$ далее станем называть подстановкой сдвига. В случае, когда $c_{n}$ лежит в $J(f)$, будем говорить, что функция $f$ является циклически замкнутой. Обозначим $D(n)=D\left(c_{n}, n\right)$ число циклически замкнутых биюнктивных функций от $n$ переменных. Воспользовавшись доказанным утверждением, получаем, что число биюнктивных функций от $n$ переменных, инвариантных относительно произвольной полноцикловой подстановки, совпадает с числом циклически замкнутых функций, то есть равно $D(n)$. Для произвольного вещественного числа $a$ наибольшее целое число, не превосходящее $a$, будем обозначать $\lfloor a\rfloor$. Обозначим также $\tau(n)$ число делителей натурального $n$ и $(k, l)$ наибольший общий делитель натуральных чисел $k$ и $l$.

Основными результатами настоящей статьи являются следующие две теоремы.

Теорема 1. Если п нечетно, то

$$
D(n)=4-\tau(n)+\sum_{k: k>0, k \mid n} 2^{\lfloor(k+2) / 2\rfloor},
$$

и если п четно, то

$$
D(n)=4-\tau(n)+\tau(n / 2)+\sum_{k: k>0, k \mid n} 2^{\lfloor(k+2) / 2\rfloor} .
$$

Теорема 2. Пусть подстановка s разлагается на $k$ независимых чиклов длины $l_{1}, \ldots, l_{k}$. Тогда

$$
D(s, n) \leqslant 7^{\sum_{1 \leqslant a<b \leqslant k}\left(l_{a}, l_{b}\right)} \prod_{i=1}^{k} D\left(l_{i}\right) .
$$

\section{2. Класс циклически замкнутых биюнктивных функций}

Будем обозначать знаком इ равенство на множестве булевых функций от $n$ переменных. Введем следующую операцию на этом множестве:

$$
f^{*}\left(x_{0}, \ldots, x_{n-1}\right) \equiv f\left(x_{0}, \ldots, x_{n-1}\right) f\left(x_{1}, \ldots, x_{n-1}, x_{0}\right) \ldots f\left(x_{n-1}, x_{0}, \ldots, x_{n-2}\right) .
$$

Отметим некоторые очевидные свойства введенной операции.

Лемма 1. Булева функция $f$ циклически замкнута тогда и только тогда, когда выполнено равенство

$$
f^{*}\left(x_{0}, \ldots, x_{n-1}\right) \equiv f\left(x_{0}, \ldots, x_{n-1}\right) .
$$

Доказательство. Если функция $f$ циклически замкнута, то подстановка $c_{n}$ лежит в группе $J(f)$ и

$$
f\left(x_{c_{n}^{k}(0)}, \ldots, x_{c_{n}^{k}(n-1)}\right) \equiv f\left(x_{0}, \ldots, x_{n-1}\right), \quad k=1,2, \ldots
$$

Отсюда следует, что

$$
\begin{aligned}
f^{*}\left(x_{0}, \ldots, x_{n-1}\right) & \equiv f\left(x_{0}, \ldots, x_{n-1}\right) f\left(x_{1}, \ldots, x_{n-1}, x_{0}\right) \ldots f\left(x_{n-1}, x_{0}, \ldots, x_{n-2}\right) \\
& \equiv f\left(x_{0}, \ldots, x_{n-1}\right) .
\end{aligned}
$$

Обратное утверждение докажем от противного. Предположим, что верно равенство

$$
f^{*}\left(x_{0}, \ldots, x_{n-1}\right) \equiv f\left(x_{0}, \ldots, x_{n-1}\right)
$$


и подстановка $c_{n}$ не лежит в группе $J(f)$. Тогда должен существовать вектор $y=\left(y_{0}, \ldots, y_{n-1}\right)$ такой, что выполнено условие

$$
f\left(y_{0}, \ldots, y_{n-1}\right) \neq f\left(y_{1}, \ldots, y_{n-1}, y_{0}\right) .
$$

Предположим, что $f\left(y_{0}, \ldots, y_{n-1}\right)=1$. Тогда $f\left(y_{1}, \ldots, y_{n-1}, y_{0}\right)=0$, и в силу (1) выполнено равенство $f^{*}\left(y_{0}, \ldots, y_{n-1}\right)=0$, что противоречит исходному предположению.

Предположим теперь, что

$$
f\left(y_{0}, \ldots, y_{n-1}\right)=0 \text {. }
$$

Тогда верно равенство

$$
f\left(y_{1}, \ldots, y_{n-1}, y_{0}\right)=1 \text {. }
$$

Положим

$$
z=\left(z_{0}, \ldots, z_{n-1}\right)=\left(y_{1}, \ldots, y_{n-1}, y_{0}\right)
$$

В этом случае

$$
\left(y_{0}, \ldots, y_{n-1}\right)=\left(z_{n-1}, z_{0}, \ldots, z_{n-2}\right)
$$

и к вектору $z$ можно применить предыдущие рассуждения и получить такое же противоречие. Лемма доказана.

Лемма 2. Для любых двух булевых функиий $f$ и $g$ справедливо равенство

$$
(f g)^{*} \equiv f^{*} g^{*}
$$

Утверждение этой леммы очевидным образом следует из коммутативности операции умножения булевых функций.

Замечание 1. Простейшим примером нетривиальных (то есть отличных от функций, тождественно равных 0 или 1) циклически замкнутых биюнктивных функций, являются функции веса единица $x_{0} \ldots x_{n-1}$ и $\bar{x}_{0} \ldots \bar{x}_{n-1}$. Из леммы 2 следует, что если ненулевая булева функщия циклически замкнута и имеет простую имплиценту длины 1 , то она совпадает с одной из двух указанных функщий.

Согласно [7] биюнктивная функция является монотонной тогда и только тогда, когда существует 2-КНФ этой функции, в которую все переменные входят только без отрицания. При этом такая 2-КНФ является одновременно сокращенной и минимальной КНФ этой функции. Аналогично, для антимонотонной биюнктивной функщии существует 2-КНФ, в которую переменные входят только с отрицаниями. Такая 2-КНФ является сокращенной и минимальной КНФ.

В соответствии с [1] мультиаффинной функцией назовем функцию, представимую в виде произведения аффинных функций.

Как показывает следующее утверждение, монотонные, антимонотонные и мультиаффинные биюнктивные функции играют главную роль в строении циклически замкнутых биюнктивных функций.

Предложение 2. Пусть $f\left(x_{0}, \ldots, x_{n-1}\right)$ - циклически замкнутая биюнктивная функция. Тогда $f$ представима в виде произведения функиий $f \equiv g h$, где $g$-мультиаффинная, $a$ $h$ - монотонная, либо антимонотонная чиклически замкнутые биюнктивные функии. 
Доказательство. Согласно предыдущему замечанию, если функция $f$ имеет простую имплиценту длины 1 , то она либо нулевая, либо совпадает с одной из двух функций $x_{0} \ldots x_{n-1}$ и $\bar{x}_{0} \ldots \bar{x}_{n-1}$. Очевидно, что в этом случае утверждение верно. Пусть теперь все простые имплиценты имеют длину, большую 1. Рассмотрим сокращенную 2-КНФ функщия $f$ и построим для нее орграф $G=(V, E)$. Поскольку простых имплицент длины 1 нет, в сокращенной КНФ все простые имплиценты имеют длину 2. В этом случае граф $G$ не имеет дуг вида $(\bar{u}, u), u \in V$. Для произвольной группы подстановок $H$ на множестве $\left\{x_{0}, \ldots, x_{n-1}\right\}$ определим группу $\tilde{H}$, действующую на множестве $V=$ $\left\{x_{0}, \ldots, x_{n-1}, \bar{x}_{0}, \ldots, \bar{x}_{n-1}\right\}$, полагая $\tilde{H}=\{\tilde{h}: h \in H\}$, где $\tilde{h}-$ расширение подстановки $h$ на множество $V$ :

$$
\tilde{h}\left(x_{i}\right)=x_{h(i)}, \quad \tilde{h}\left(\bar{x}_{i}\right)=\bar{x}_{h(i)}, \quad i=0,1, \ldots, n-1 .
$$

По теореме о группах инерции биюнктивных функций (см. [7])

$$
J(f)=\left\{h \in S_{n}: \tilde{h} \in \text { Aut } G\right\} .
$$

Так как подстановка $c_{n}$ лежит в группе $J(f)$, подстановка $\tilde{c}_{n}$ лежит в группе Aut $G$. Отсюда, в силу свойств графов 2-КНФ, следует, что все вершины графа $G$ вида $x_{i}$, $i=1, \ldots, n-1$, имеют одинаковые полустепени захода и исхода $\left(r^{+}, r^{-}\right)$, а вершины вида $\bar{x}_{i}, i=1, \ldots, n-1$, имеют одинаковые полустепени захода и исхода $\left(r^{-}, r^{+}\right)$. Возможны два варианта.

- Все вершины одного из множеств $X$ или $\bar{X}$ - начальные вершины. Тогда либо выполнены условия $r^{+}=0$ и $r^{-}>0$, либо выполнены условия $r^{+}>0$ и $r^{-}=0$. В первом случае функция $f$, очевидно, монотонна, во втором антимонотонна.

- Каждая вершина является проходной и лежит на некотором цикле.

Пусть $E_{1}$ - множество дуг графа $G$, не входящих в сильно связные компоненты, $E_{2}=E \backslash E_{1}$. Представим граф $G$ в виде суммы $G=G_{1}+G_{2}$ графов $G_{1}=\left(V, E_{1}\right)$ и $G_{2}=\left(V, E_{2}\right)$. Оба графа, очевидно, являются графами 2-КНФ биюнктивных функций. Пусть граф $G_{1}$ есть граф 2-КНФ функции $f_{1}$, a $G_{2}-$ функции $f_{2}$. Из того, что подстановка $\tilde{c}_{n}$ лежит в группе Aut $G$ следует, что выполнены условия $\tilde{c}_{n} \in \operatorname{Aut} G_{1}, \tilde{c}_{n} \in \operatorname{Aut} G_{2}$. Следовательно, обе построенные функции циклически замкнуты. При этом граф $G_{1}$ не имеет проходных вершин, поскольку в этом случае все его вершины будут проходными и, следовательно, он имеет сильно связные компоненты, что противоречит его построению. Тогда, по предыдущей части доказательства, функщия $f_{1}$ является либо монотонной, либо антимонотонной.

Рассмотрим граф $G_{2}$. По построению этот граф не имеет дуг, не лежащих в сильно связных компонентах. Следовательно, все его сильно связные компоненты изолированы и функция $f_{2}$ является мультиаффинной. Утверждение доказано.

Заметим, что представление биюнктивной функции в виде произведения, указанное в доказанном утверждении, не является однозначным. Однако становится понятным, что для описания циклически замкнутых биюнктивных функций необходимо более глубокое изучение классов монотонных, антимонотонных и мультиаффинных биюнктивных функций.

Множество выполняющих векторов булевой функщии $f\left(x_{0}, \ldots, x_{n-1}\right)$ обозначим чеpeз $N(f)$, то есть

$$
N(f)=\left\{\left(u_{0}, \ldots, u_{n-1}\right): f\left(u_{0}, \ldots, u_{n-1}\right)=1\right\}
$$


Докажем вспомогательную лемму.

Лемма 3. Справедливы равенства

$$
\begin{gathered}
\left(x_{0} \vee x_{i}\right)^{*}=\left(x_{0} \vee x_{n-i}\right)^{*}, \quad i=1,2, \ldots, n-1 ; \\
\prod_{i=1}^{l}\left(x_{0} \oplus x_{r_{1}} \oplus 1\right)^{*}=\left(x_{0} \oplus x_{d} \oplus 1\right)^{*}
\end{gathered}
$$

где $d=\left(r_{1}, \ldots, r_{k}, n\right)-$ наибольший общий делитель чисел $r_{1}, \ldots, r_{k}, n$.

Если $n$ четно, $d_{j}=(j, n / 2)$ и $j / d_{j}$ нечетно, то справедливо равенство

$$
\left(x_{0} \oplus x_{j}\right)^{*}=\left(x_{0} \oplus x_{d_{j}}\right)^{*} \text {. }
$$

Во всех остальных случаях

$$
\left(x_{0} \oplus x_{j}\right)^{*} \equiv 0
$$

Кроме того, если п четно, $d_{i j}=(i, j, n / 2)$, то справедливы равенства

$$
\left(x_{0} \oplus x_{i}\right)^{*}\left(x_{0} \oplus x_{j}\right)^{*}=\left(x_{0} \oplus x_{d_{i j}}\right)^{*},
$$

если числа $i / d_{i j}, j / d_{i j}$ нечетны, $u$

$$
\left(x_{0} \oplus x_{i}\right)^{*}\left(x_{0} \oplus x_{j}\right)^{*} \equiv 0,
$$

если среди чисел $i / d, j / d$ есть четное число.

Кроме того, справедливы равенства

$$
\left(x_{0} \oplus x_{i}\right)^{*}\left(x_{0} \oplus x_{j} \oplus 1\right)^{*}=\left(x_{0} \oplus x_{d_{i j}}\right)^{*},
$$

если число $j / d_{i j}$ четно, $и$

$$
\left(x_{0} \oplus x_{i}\right)^{*}\left(x_{0} \oplus x_{j} \oplus 1\right)^{*} \equiv 0,
$$

если число $j / d_{i j}$ нечетно.

Доказательство. Докажем равенство (2). По определению

$$
\begin{aligned}
\left(x_{0} \vee x_{i}\right)^{*} & =\prod_{k=0}^{n-1}\left(x_{k} \vee x_{(k+i) \bmod n}\right), \\
\left(x_{0} \vee x_{n-i}\right)^{*} & =\prod_{k=0}^{n-1}\left(x_{k} \vee x_{(k+n-i) \bmod n}\right)=\prod_{k=0}^{n-1}\left(x_{k} \vee x_{(k-i) \bmod n}\right) .
\end{aligned}
$$

Очевидно, что множества неупорядоченных пар

$$
\begin{array}{ll}
N_{1}=\{\{k,(k+i) \bmod n\}, & k=0,1, \ldots, n-1\}, \\
N_{2}=\{\{k,(k-i) \bmod n\}, & k=0,1, \ldots, n-1\}
\end{array}
$$

совпадают. Таким образом, равенство (2) доказано. 
Докажем равенство (3). Рассмотрим функции

$$
\begin{aligned}
& f\left(x_{0}, \ldots, x_{n-1}\right)=\prod_{i=1}^{l}\left(x_{0} \oplus x_{r_{1}} \oplus 1\right)^{*}, \\
& g\left(x_{0}, \ldots, x_{n-1}\right)=\left(x_{0} \oplus x_{d} \oplus 1\right)^{*}
\end{aligned}
$$

Так как $d=\left(r_{1}, \ldots, r_{l}, n\right)$, найдутся такие целые числа $u_{1}, \ldots, u_{l+1}$, что верно равенство

$$
d=u_{1} r_{1}+\ldots+u_{l} r_{l}+u_{l+1} n .
$$

Тогда, если вектор $\left(a_{0}, \ldots, a_{n-1}\right)$ лежит в множестве $N(f)$, то

$$
\begin{aligned}
a_{k} & =a_{\left(k+r_{1}\right) \bmod n}=a_{\left(k+u_{1} r_{1}\right) \bmod n} \\
& =a_{\left(k+u_{1} r_{1}+r_{2}\right) \bmod n}=\ldots=a_{\left(k+u_{1} r_{1}+\ldots+u_{l} r_{l}\right) \bmod n} \\
& =a_{\left(k+u_{1} r_{1}+\ldots+u_{l} r_{l}+u_{l+1} n\right) \bmod \dot{n} .}
\end{aligned}
$$

Отсюда следует, что $N(f) \subset N(g)$. Обратно, поскольку число $d$ делит числа $r_{1}, \ldots, r_{l}$, очевидно, что $N(g) \subset N(f)$. Равенство (3) доказано.

Перейдем к (4) и (5). Пусть $f=\left(x_{0} \oplus x_{j}\right)^{*}$. Заметим, что если множество $N(f)$ не пусто, то для любого вектора $\left(a_{0}, \ldots, a_{n-1}\right)$ из $N(f)$ и любого $k=0,1, \ldots, n-1$ выполнено условие $a_{k} \neq a_{(k+j) \bmod n}$. Отсюда следует, что для любого вектора $\left(a_{0}, \ldots, a_{n-1}\right)$ из множества $N(f)$ и для любого $k$ такого, что $0 \leqslant k \leqslant n-1$, справедливо равенство $a_{k}=a_{(k+2 j) \bmod n}$. Следовательно, функция $\left(x_{0} \oplus x_{d^{\prime}} \oplus 1\right)^{*}$, где $d^{\prime}=(2 j, n)$, удовлетворяет равенству

$$
f\left(x_{0}, \ldots, x_{n-1}\right)\left(x_{0} \oplus x_{d^{\prime}} \oplus 1\right)^{*} \equiv f\left(x_{0}, \ldots, x_{n-1}\right) .
$$

Если $n$ нечетно, то $d^{\prime}=(2, j, n)=(j, n)$. В этом случае, поскольку выполнено равенство (10) и число $d^{\prime}$ делит $j$, получаем, что для любого вектора $\left(a_{0}, \ldots, a-n-1\right)$ из множества $N(f)$ выполнено условие $a_{k}=a_{(k+j) \bmod n}$. Но на таких векторах функщия $f=\left(x_{0} \oplus x_{j}\right)^{*}$ принимает значение 0 . Из полученного противоречия следует, что $f \equiv 0$.

Теперь рассмотрим случай, когда $n$ четно. Если $j / d_{j}$ четно, то число $d^{\prime}=2 d_{j}$ делит число $j$. В этом случае выполняется равенство $d^{\prime}=(j, n)$, из которого, как показано выше, следует, что множество $N(f)$ пусто. Равенство (5) доказано.

Пусть теперь $j / d_{j}$ нечетно. Положим $g=\left(x_{0} \oplus x_{d}\right)^{*}$. Покажем, что $f \equiv g$. Поскольку $d_{j}$ делит $j$ и $j / d_{j}$ нечетно, справедливо включение $N(g) \subset N(f)$. Докажем обратное включение. Введем множества

$$
\begin{aligned}
M & =\{(2 k+1) j \bmod n, k=0,1, \ldots\}, \\
L & =\{(2 k+1) d \bmod n, k=0,1, \ldots\} .
\end{aligned}
$$

В этих обозначениях вектор $\left(a_{0}, \ldots, a_{n-1}\right)$ лежит в множестве $N(f)$ тогда и только тогда, когда для любого $u$ из множества $M$ и для любого $l$ такого, что $0 \leqslant l \leqslant n-1$, выполняется равенство $a_{l}=a_{l+u} \oplus 1$. Аналогичное утверждение верно для векторов $\left(b_{0}, \ldots, b_{n-1}\right) \in N(g)$ с заменой множества $M$ на множество $L$. Следовательно, поскольку выполнено включение $N(g) \subset N(f)$, должно выполняться и включение $L \subset M$. В силу очевидных соображений

$$
|M|=\frac{n}{(2 j, n)}=\frac{n}{2 d_{j}}=|L| .
$$


Поэтому $M=L$. Из этого следует равенство $f \equiv g$. Формула (4) доказана.

Перейдем к равенствам (6) и (7). Пусть $n$ четно. Без ограничения общности можно считать, что числа $i$ и $j$ делят число $n / 2$. Тогда очевидно равенство $d_{i j}=(i, j)$. Положим

$$
f=\left(x_{0} \oplus x_{i}\right)^{*}\left(x_{0} \oplus x_{j}\right)^{*}, \quad g=\left(x_{0} \oplus x_{d_{i j}}\right)^{*}
$$

и $a=i / d_{i j}, b=j / d_{i j}$. Очевидно, что числа $a$ и $b$ взаимно просты. Заметим, что оба введенных числа не могут быть четными одновременно. Пусть числа $a$ и $b$ нечетны. Тогда для любого вектора $\left(y_{0}, \ldots, y_{n-1}\right)$ из множества $N(g)$ выполняются равенства

$$
\begin{aligned}
& y_{l}=y_{\left(l+d_{i j}\right) \bmod n} \oplus 1=y_{\left(l+a d_{i j}\right) \bmod n} \oplus 1=y_{l+i} \oplus 1, \quad l=0,1, \ldots, n-1,
\end{aligned}
$$

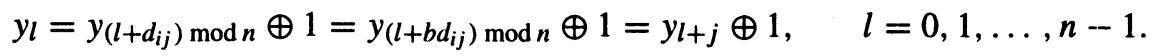

Отсюда следует, что вектор ( $\left.y_{0}, \ldots, y_{n-1}\right)$ принадлежит множеству $N(f)$ и выполняется включение $N(g) \subset N(f)$. Покажем, что эти множества совпадают. Предположим, что в $N(f)$ содержится вектор $\left(y_{0}, \ldots, y_{n-1}\right)$ такой, что $g\left(y_{0}, \ldots, y_{n-1}\right)=0$. Это означает, что для некоторого числа $l \in\{0,1, \ldots, n-1\}$ справедливо равенство $y_{l}=y_{\left(l+d_{i j}\right) \bmod n}$. Для краткости записи будем считать, что $l=0$. Поскольку $d_{i j}=(i, j)$, найдутся такие числа $u$ и $v$, что выполнено равенство $u i+v j=d_{i j}$. Тогда

$$
d_{i j}=u i+v j=u a d_{i j}+v b d_{i j}=(u a+v b) d_{i j},
$$

откуда следует, что $u a+v b=1$. Так как $a$ и $b$ нечетны, числа $u$ и $v$ должны иметь разную четность. Предположим, что $u$ четно, а $v$ нечетно. Положим $u^{\prime}=u \bmod n, v^{\prime}=v \bmod n$. Поскольку число $n$ четно, то число $u^{\prime}$ четно, а $v^{\prime}$ нечетно, и верно равенство $u^{\prime} a+v^{\prime} b=$ $1 \bmod n$. Тогда верна цепочка равенств

$$
y_{0}=y_{i} \oplus 1=\ldots=y_{\left(u^{\prime} i\right) \bmod n}=y_{\left(u^{\prime} i+j\right) \bmod n} \oplus 1=\ldots=y_{\left(u^{\prime} i+v^{\prime} l\right) \bmod n} \oplus 1=y_{d} \oplus 1,
$$

что противоречит исходному предположению. Следовательно, множества $N(f)$ и $N(g)$ совпадают и равенство (6) верно.

Предположим теперь, что $a$ четно, а $b$ нечетно. Поскольку $d_{i j}=(i, j)$, найдутся такие числа $u$ и $v$, что справедливо равенство $u i+v j=d_{i j}$. Тогда

$$
d_{i j}=u i+v j=u a d_{i j}+v b d_{i j}=(u a+v b) d_{i j},
$$

откуда следует, что $u a+v b=1$. Так как $a$ четно, а $b$ нечетно, то $v$ нечетно. Положим $u^{\prime}=u \bmod n, v^{\prime}=v \bmod n$. Поскольку число $n$ четно, то $v^{\prime}$ нечетно и верно равенство $u^{\prime} a+v^{\prime} b=l \bmod n$. Тогда для любого вектора $\left(y_{0}, \ldots, y_{n-1}\right)$ из множества $N(f)$ справедливы равенства

$$
y_{l}=y_{\left(u^{\prime} i+v^{\prime} j\right) \bmod n} \oplus 1=y_{\left(l+d_{i j}\right) \bmod n} \oplus 1, \quad l=0,1, \ldots, n-1 .
$$

Следовательно, поскольку $а$ четно, будут выполняться равенства

$$
y_{l}=y_{\left(l+a d_{i j}\right) \bmod n}=y_{(l+i) \bmod n}, \quad l=0,1, \ldots, n-1,
$$

что ведет к противоречию с определением функции $f$. Следовательно, множество $N(f)$ пусто и выполнено равенство (7).

Докажем соотношения (8) и (9). Положим

$$
f=\left(x_{0} \oplus x_{i}\right)^{*}\left(x_{0} \oplus x_{j} \oplus 1\right)^{*} .
$$


При этом, согласно предыдущим равенствам, можно считать, что числа $2 i$ и $j$ делят число $n$. Пусть $d_{i j}=(i, j)$. Положим $a=i / d_{i j}, b=j / d_{i j}$. Тогда $(a, b)=1$ и иa $+v b=1$. Кроме того, числа $2 a$ и $b$ делят число $n$. Положим $f_{1}=\left(x_{0} \oplus x_{i}\right)^{*}, f_{2}=\left(x_{0} \oplus x_{j} \oplus 1\right)^{*}$. Тогда верно равенство $N(f)=N\left(f_{1}\right) \cap N\left(f_{2}\right)$. При этом

$$
\begin{aligned}
& N\left(f_{1}\right)=\left\{\left(z_{0}, \ldots, z_{n-1}: z_{l}=z_{(l+i) \bmod n} \oplus 1, l=0,1, \ldots, n-1\right)\right\}, \\
& N\left(f_{2}\right)=\left\{\left(z_{0}, \ldots, z_{n-1}: z_{l}=z_{(l+j) \bmod n}, l=0,1, \ldots, n-1\right)\right\} .
\end{aligned}
$$

Рассмотрим случай, когда $b$ нечетно. В этом случае, поскольку $a b d_{i j}=a j=b i$ и $\boldsymbol{n}$ четно, найдутся такие числа $c, r \in\{0,1, \ldots, n-1\}$, что $r j=c i \bmod n$, причем число $c$ нечетно. Тогда для всякого вектора $\left(z_{0}, \ldots, n-1\right) \in N\left(f_{1}\right)$ выполнено равенство

$$
z_{l}=z_{(l+b i) \bmod n} \oplus 1=z_{(l+r j) \bmod n} \oplus 1 .
$$

Значит, $f_{2}\left(z_{0}, \ldots, z_{n-1}\right)=0$, и следовательно, $f \equiv 0$. Равенство (9) доказано.

Пусть теперь $b$ четно. Тогда, поскольку $(a, b)=1$, число $a$ нечетно. В этом случае из того, что $u a+v b=1$ следует, что $u$ нечетно. Положим $g\left(x_{0} \oplus x_{d_{i j}}\right)^{*}$. Тогда очевидны включения

$$
N(g) \subset N\left(f_{1}\right), \quad N(g) \subset N\left(f_{2}\right) .
$$

Следовательно, $N(g) \subset N(f)$.

Докажем обратное включение. Пусть $\left(z_{0}, \ldots, z_{n-1}\right) \in N(f)$. Тогда для любого $l=0,1, \ldots, n-1$ верны равенства

$$
z_{l}=z_{(l+v j) \bmod n}=z_{(l+u i+v j) \bmod n \oplus 1 .}
$$

Значит, $\left(z_{0}, \ldots, z_{n-1}\right) \in N(g)$. Отсюда следует, что $f \equiv g$ и соотношение (8) доказано. Лемма доказана.

Пользуясь доказанной леммой, мы можем описать строение классов монотонных, антимонотонных и мультиаффинных циклически замкнутых биюнктивных функщий и, в частности, подсчитать мощности этих классов.

Предложение 3. Пусть $f$ является циклически замкнутой биюнктивной функцией, отличной от константы и не имеющей элементарных импличент длины 1. Тогда:

- если $f$ монотонна, то ее 2-КНФ представляется в виде

$$
\left(x_{0} \vee x_{i_{1}}\right)^{*} \ldots\left(x_{0} \vee x_{i_{k}}\right)^{*},
$$

где $1 \leqslant k \leqslant\lfloor(n-1) / 2\rfloor, 0<i_{1}<\ldots<i_{k} \leqslant\lfloor(n-1) / 2\rfloor$. Функция $f$ однозначно определяет набор чисел $k, i_{1}, \ldots, i_{k}$ (то есть представление (11) единственно);

- если $f$ антимонотонна, то ее 2-КНФ представляется в виде

$$
\left(\bar{x}_{0} \vee \bar{x}_{i_{1}}\right)^{*} \ldots\left(\bar{x}_{0} \vee \bar{x}_{i_{k}}^{*}\right) \text {, }
$$

где $1 \leqslant k \leqslant\lfloor(n-1) / 2\rfloor, 0<i_{1}<\ldots<i_{k} \leqslant\lfloor(n-1) / 2\rfloor$. Функция $f$ однозначно определяет набор чисел $k, i_{1}, \ldots, i_{k}$;

- если $f$ мультиаффинна, то в случае, когда $f(0, \ldots, 0)=f(1, \ldots, 1)=1$, существует единственное число $j$, делящее $n$, такое, что справедливо равенство

$$
f\left(x_{0}, \ldots, x_{n-1}\right)=\left(x_{0} \oplus x_{j} \oplus 1\right)^{*} ;
$$


- если $f(0, \ldots, 0) f(1, \ldots, 1)=0$, то число п четно и существует единственное число $j$ такое, что число $2 j$ делит $n$ и справедливо равенство

$$
f\left(x_{0}, \ldots, x_{n-1}\right)=\left(x_{0} \oplus x_{j}\right)^{*} .
$$

Доказательство. Пусть задана монотонная циклически замкнутая биюнктивная функщия $f\left(x_{0}, \ldots, x_{n-1}\right)$. Из монотонности следует, что ее 2-КНФ является сокращенной (у монотонной биюнктивной функщии существует ровно одна 2-КНФ). Поскольку $f$ циклически замкнута, в силу леммы 2 справедливо равенство

$$
f\left(x_{0}, \ldots, x_{n-1}\right)=\prod_{i=1}^{t}\left(x_{s_{i, 1}} \vee x_{s_{i, 2}}\right)^{*} .
$$

Будем считать, что $s_{i, 1}<s_{i, 2}, i=1, \ldots, t$. Положим $k_{i}=s_{i, 2}-s_{i, 1}, i=1, \ldots, t$. Очевидно равенство

$$
f\left(x_{0}, \ldots, x_{n-1}\right)=\prod_{i=1}^{t}\left(x_{0} \vee x_{k_{i}}\right)^{*} .
$$

Удалим из правой части (14) некоторые сомножители так, чтобы оставшиеся функции $\left(x_{0} \vee x_{k_{i}}\right)^{*}$ были различны. Пользуясь равенством (2), добьемся того, чтобы все значения $k_{i}$ удовлетворяли условию $k_{i} \leqslant\lfloor(n-1) / 2\rfloor$. Формула (11) доказана.

Осталось доказать, что функщия $f$ однозначно определяет набор $k, i_{1}, \ldots, i_{k}$. Проведем доказательство от противного. Предположим, что существует другой набор $k^{\prime}, i_{1}^{\prime}, \ldots, i_{k^{\prime}}^{\prime}$, также реализующий функцию $f$. Но тогда функция $\left(x_{0} \vee x_{i_{1}}\right)$ является элементарной имплицентой функции $f$, так как

$$
f=\left(x_{0} \vee x_{i_{1}^{\prime}}\right)^{*} \ldots\left(x_{0} \vee x_{i_{k^{\prime}}^{\prime}}\right)^{*} .
$$

Поскольку у функщия $f$ нет элементарных имплицент длины 1 , то $\left(x_{0} \vee x_{i_{1}^{\prime}}\right)$ является простой имплищентой. Следовательно, поскольку представление (14) является также и сокращенной КНФ, сомножитель $\left(x_{0} \vee x_{i_{1}^{\prime}}\right)$ присутствует в формуле (14). По лемме 2 в правой части (14) есть сомножитель $\left(x_{0} \vee x_{i_{1}^{\prime}}\right)^{*}$, то есть при некотором $j \in\{1, \ldots, r\}$ выполнено равенство $i_{1}^{\prime}=i_{j}$. Продолжая таким образом, получаем, что $\left\{i_{1}^{\prime}, \ldots, i_{k^{\prime}}^{\prime}\right\}$ является подмножеством $\left\{i_{1}, \ldots, i_{k}\right\}$. Поменяв местами $k, i_{1}, \ldots, i_{k}$ и $k^{\prime}, i_{1}^{\prime}, \ldots, i_{k^{\prime}}^{\prime}$ и проведя те же рассуждения, получаем, что $\left\{i_{1}, \ldots, i_{k}\right\}$ является подмножеством $\left\{i_{1}^{\prime}, \ldots, i_{k^{\prime}}^{\prime}\right\}$. Таким образом, $k=k^{\prime}$ и $\left\{i_{1}, \ldots, i_{k}\right\}=\left\{i_{1}^{\prime}, \ldots, i_{k^{\prime}}^{\prime}\right\}$.

Для антимонотонных биюнктивных циклически замкнутых функций утверждение доказывается аналогично.

Пусть $f$ - циклически замкнутая мультиаффинная биюнктивная функция, отличная от константы и не имеющая имплицент длины 1. Функцию $f$ можно записать в виде

$$
f\left(x_{0}, \ldots, x_{n-1}\right)=\prod_{p=1}^{t}\left(x_{s_{p 1}} \oplus x_{s_{p 2}} \oplus 1\right) \prod_{q=1}^{r}\left(x_{w_{q 1}} \oplus x_{w_{q 2}}\right) \text {. }
$$

Поскольку $f$ циклически замкнута, то

$$
f\left(x_{0}, \ldots, x_{n-1}\right)=\prod_{p=1}^{t}\left(x_{0} \oplus x_{k_{p}} \oplus 1\right)^{*} \prod_{q=1}^{r}\left(x_{0} \oplus x_{l_{q}}\right)^{*} .
$$


Пользуясь тождествами (3)-(9), получаем, что функцию $f$, записанную равенством (15), можно привести ровно к одному из двух вариантов, с правой частью вида (12) или с правой частью вида (13). Заметим, что если

$$
f(0, \ldots, 0)=f(1, \ldots 1)=1,
$$

то функция $f$ запишется в виде (12), в противном случае в виде (13).

Осталось доказать, что такое представление единственно. Пусть выполнены равенства $f(0, \ldots, 0)=f(1, \ldots, 1)=1$ и существует такое число $j^{\prime}$, не равное $j$ и делящее $n$, что выполнено соотношение

$$
f\left(x_{0}, \ldots, x_{n-1}\right)=\left(x_{0} \oplus x_{j^{\prime}} \oplus 1\right)^{*} .
$$

Из равенства (3) следует соотношение

$$
f\left(x_{0}, \ldots, x_{n-1}\right)=\left(x_{0} \oplus x_{d} \oplus 1\right)^{*},
$$

где $d=\left(j, j^{\prime}\right)$. Таким образом, выполнено равенство

$$
\left(x_{0} \oplus x_{j} \oplus 1\right)^{*}=\left(x_{0} \oplus x_{d} \oplus 1\right)^{*},
$$

причем $d$ делит $j$ и $d<j$.

Определим вектор $\left(y_{0}, \ldots, y_{n-1}\right)$ такой, что $y_{i}=0$, если $\lfloor i / j\rfloor$ четно, и $y_{i}=1$, если $\lfloor i / j\rfloor$ нечетно. Функция $\left(x_{0} \oplus x_{j} \oplus 1\right)^{*}$ принимает значение 1 , а функция $\left(x_{0} \oplus x_{d} \oplus 1\right)^{*}$ значение 0.

В случае, когда $f(0, \ldots, 0) \neq f(1, \ldots, 1)$, доказательство проводится аналогично. Утверждение доказано.

Для натурального числа $n$ пусть $\tau(n)$ - число делителей числа $n$. Сформулируем следствие из доказанного утверждения.

Следствие 1. Число биюнктивных монотонных (антимонотонных) чиклически замкнутых функций от $n$ переменных, отличных от константы и не имеющих имплицент длины 1 , равно $2^{\lfloor(n-1) / 2\rfloor-1}$.

Число биюнктивных мультиаффинных чиклически замкнутых функций от $n$ переменных, отличных от константы и не имеючих имплицент длины 1, равно $\tau(n)$, когда $n$ нечетно, и $\tau(n)+\tau(n / 2)$, когда $n$ четно.

Доказательство. Биюнктивных монотонных (антимонотонных) циклически замкнутых функиий от $n$ переменных, отличных от константы и не имеющих имплицент длины 1 , столько же, сколько непустых подмножеств в множестве целых чисел от 1 до $\lfloor(n-1) / 2\rfloor$. Это число равно $2^{\lfloor(n-1) / 2\rfloor}-1$.

В случае, когда $n$ нечетно, число $2 j$ не делит $n$. Следовательно, все мультиаффинные функщии имеют вид (12). Из единственности представления (12) получаем, что число различных функций совпадает с числом делителей числа $n$, то есть с $\tau(n)$. В случае, когда $n$ четно, добавляется число $\tau(n / 2)$, равное числу различных функций вида (13). Следствие доказано.

Для натуральных чисел $i$ и $k$ определим функцию

$$
\varphi_{k}(i)= \begin{cases}i \bmod k, & \text { если } i \bmod k \leqslant k / 2, \\ k-(i \bmod k) & \text { в противном случае. }\end{cases}
$$

Связь между классами монотонных (антимонотонных) и мультиаффинных циклически замкнутых биюнктивных функций показывает следующая лемма.

2 Дискретная математика, т.14 №3 
Лемма 4. Справедливы равенства

$$
\left(x_{0} \vee x_{i}\right)^{*}\left(x_{0} \oplus x_{j}\right)^{*} \equiv\left(x_{0} \oplus x_{i}\right)^{*}\left(x_{0} \oplus x_{j}\right)^{*} .
$$

Кроме того,

$$
\begin{aligned}
\left(x_{0} \vee x_{i_{1}}\right)^{*}\left(x_{0} \vee x_{i_{2}}\right) \ldots\left(x_{0} \vee x_{i_{r}}\right)^{*} & \left(x_{0} \oplus x_{k} \oplus 1\right)^{*} \\
& \equiv\left(x_{0} \vee x_{\varphi_{k}\left(i_{1}\right)}\right)^{*} \ldots\left(x_{0} \vee x_{\varphi_{k}\left(i_{r}\right)}\right)^{*}\left(x_{0} \oplus x_{k} \oplus 1\right)^{*} .
\end{aligned}
$$

Функиии

$$
\begin{aligned}
& \left(x_{0} \vee x_{i_{1}}\right)^{*}\left(x_{0} \vee x_{i_{2}}\right)^{*} \ldots\left(x_{0} \vee x_{i_{r}}\right)^{*}\left(x_{0} \oplus x_{k} \oplus 1\right)^{*}, \\
& \left(x_{0} \vee x_{j_{1}}\right)^{*}\left(x_{0} \vee x_{j_{2}}\right)^{*} \ldots\left(x_{0} \vee x_{j_{s}}\right)^{*}\left(x_{0} \oplus x_{d} \oplus 1\right)^{*},
\end{aligned}
$$

где для первой функции $k \mid n u 0<i_{1}, \ldots<i_{r} \leqslant k / 2$, a для второй $d \mid n u$ $0<j_{1}<\ldots<j_{s} \leqslant d / 2$, совпадают тогда и только тогда, когда $k=d u$ совпадают множества $\left\{i_{1}, \ldots, i_{r}\right\} u\left\{j_{1}, \ldots, j_{s}\right\}$.

Все указанные свойства остаются верными при навешивании отрицаний на все переменные в формулах (16)-(18).

Доказательство. Докажем равенство (16). Введем функции $f \equiv\left(x_{0} \vee x_{i}\right)^{*}$ и $g \equiv\left(x_{0} \oplus x_{j}\right)^{*}$. Пусть вектор $\left(a_{0}, \ldots, a_{n-1}\right)$ лежит в множестве $N(f g)$. Тогда справедливо равенство $a_{0} \vee a_{i}=1$. Если выполнено условие $a_{0}=a_{i}=1$, то из равенства $a_{0}=1$ следует равенство $a_{j}=0$, а из равенства $a_{i}=1$ следует, что $a_{(i+j) \bmod n}=0$. Но тогда $a_{j} \vee a_{(i+j) \bmod n}=0$, то есть $f\left(a_{0}, \ldots, a_{n-1}\right)=0$. Получили противоречие с тем, что вектор $\left(a_{0}, \ldots, a_{n-1}\right)$ лежит в множестве $N(f g)$. Следовательно, выполнено условие $a_{0} \neq a_{i}$. Аналогичными рассуждениями легко показать, что если вектор $\left(a_{0}, \ldots, a_{n-1}\right)$ лежит в множестве $N(f g)$, то для любых $k, l=0,1, \ldots, n-1$ выполнено равенство

$$
a_{(l+k i) \bmod n} \oplus a_{(l+(k+1) i) \bmod n}=1 .
$$

Отсюда следует, что

$$
f g \equiv\left(x_{0} \oplus x_{i}\right)^{*} g \equiv\left(x_{0} \oplus x_{i}\right)^{*}\left(x_{0} \oplus x_{j}\right)^{*} .
$$

Равенство (16) доказано.

Перейдем к доказательству равенства (17). Введем обозначения

$$
\begin{aligned}
f_{1} & \equiv\left(x_{0} \vee x_{i_{1}}\right)^{*} \ldots\left(x_{0} \vee x_{i_{r}}\right)^{*}, \\
f_{2} & \equiv\left(x_{0} \vee x_{\varphi_{k}\left(i_{1}\right)}\right)^{*} \ldots\left(x_{0} \vee x_{\varphi_{k}\left(i_{r}\right)}\right)^{*}, \\
g & \equiv\left(x_{0} \oplus x_{k} \oplus 1\right)^{*} .
\end{aligned}
$$

Пусть $\left(a_{0}, \ldots, a_{n-1}\right) \in N\left(f_{1} g\right)$. Тогда $g\left(a_{0}, \ldots, a_{n-1}\right)=1$ и $f_{1}\left(a_{0}, \ldots, a_{n-1}\right)=1$. Покажем, что $f_{2}\left(a_{0}, \ldots, a_{n-1}\right)=1$. Если в векторе $\left(a_{0}, \ldots, a_{n-1}\right)$ нет нулевых координат, то очевидно, что $f_{2}\left(a_{0}, \ldots, a_{n-1}\right)=1$. В противном случае необходимо и достаточно доказать, что если $a_{l}=0$, то для любого $s=1, \ldots, r$ выполнены условия $a_{\left(l-\varphi_{k}\left(i_{s}\right)\right) \bmod n}=1$, $a_{\left(l+\varphi_{k}\left(i_{s}\right)\right) \bmod n}=1$. Заметим, что, так как $f_{1}\left(a_{0}, \ldots, a_{n-1}\right)=1$, то $a_{\left(l+i_{s}\right) \bmod n}=1$. Кроме того, из равенства $g\left(a_{0}, \ldots, a_{n-1}\right)=1$ следует, что $a_{\left(l+i_{s}+t k\right) \bmod n}=1$ для любого целого $t$. Из определения функции $\varphi_{k}$ следует, что для любого $i=0,1, \ldots, n-1$ существует такое целое $q$, что $\varphi_{k}(i)+q k=i$, то есть при некотором значении $t$ выполнено равенство

$$
a_{\left(l+i_{s}+t k\right) \bmod n}=a_{\left(l+\varphi_{k}\left(i_{s}\right)\right) \bmod n}=1 .
$$


Аналогично показывается, что $a_{\left(l-i_{s}-t k\right) \bmod n}=a_{\left(l-\varphi_{k}\left(i_{s}\right)\right) \bmod n}=1$. Таким образом, $N\left(f_{1} g\right) \subset N\left(f_{2} g\right)$. Обратное включение доказывается таким же образом с учетом того, что $\varphi_{k}(i)=i-q k$.

Докажем (18). Введем обозначения

$$
\begin{array}{ll}
g_{1} \equiv\left(x_{0} \oplus x_{k} \oplus 1\right)^{*}, & g_{2} \equiv\left(x_{0} \oplus x_{d} \oplus 1\right)^{*}, \\
f_{1} \equiv\left(x_{0} \vee x_{i_{1}}\right)^{*}\left(x_{0} \vee x_{i_{2}}\right)^{*} \ldots\left(x_{0} \vee x_{i_{r}}\right)^{*}, & f_{2} \equiv\left(x_{0} \vee x_{j_{1}}\right)^{*}\left(x_{0} \vee x_{j_{2}}\right)^{*} \ldots\left(x_{0} \vee x_{j_{s}}\right)^{*},
\end{array}
$$

причем $0<i_{1}<\ldots<i_{r} \leqslant k / 2,0<j_{1}<\ldots<j_{s} \leqslant d / 2, k \mid r$ и $d \mid n$.

Докажем, что $k=d$. Без ограничения общности будем считать, что $d \geqslant k$. Тогда для вектора $\left(a_{0}, \ldots, a_{n-1}\right)$ такого, что для любого целого $t$ выполнено условие $a_{t k \bmod n}=0$ и все остальные значения $a_{i}=1$, функция $f_{1} g_{1}$ принимает значение 1 , так как все значения $i_{q}, q=1, \ldots, r$, удовлетворяют неравенству $i_{q} \leqslant k / 2<k$. Тогда, если вектор $\left(a_{0}, \ldots, a_{n-1}\right)$ лежит в множестве $N\left(f_{2} g_{2}\right)$, то $a_{0} \oplus a_{d}=0$. Далее, поскольку $a_{0}=0$, то $a_{d}=0$. Следовательно, $d=t k \bmod n$. Учитывая, что $k$ делит $n$, получаем, что $d$ делит $k$. Из неравенства $d \geqslant k$ следует, что $d=k$.

Пусть теперь выполнено соотношение $g \equiv g_{1} \equiv g_{2}$. Покажем, что если множества $\left\{i_{1}, \ldots, i_{r}\right\}$ и $\left\{j_{1}, \ldots, j_{s}\right\}$ не совпадают, то не совпадают и функщии $f_{1} g$ и $f_{2} g$. Без ограничения общности считаем, что $j_{1} \notin\left\{i_{1}, \ldots, i_{r}\right\}$ и $j_{1} \neq k / 2$. Построим вектор $\left(a_{0}, \ldots, a_{n-1}\right)$ такой, что для любого целого $t$ выполнены уссловия $a_{t k}=0$ и $a_{j_{1}+t k}=0$, а все остальные значения $a_{i}=1$. Очевидно, что $\left(a_{0}, \ldots, a_{n-1}\right) \notin N\left(f_{2} g\right)$, поскольку $a_{0} \vee a_{j_{1}}=0$. Докажем теперь, что $\left(a_{0}, \ldots, a_{n-1}\right) \in N\left(f_{1} g\right)$. Предположим противное. Поскольку по построению вектора $\left(a_{0}, \ldots, a_{n-1}\right)$ выполнено условие $a_{l}=a_{(l+k) \bmod n}, 0 \leqslant l \leqslant n-1$, то справедливо равенство $g\left(a_{0}, \ldots, a_{n-1}\right)=1$. Следовательно, $f\left(a_{0}, \ldots, a_{n-1}\right)=0$, то есть существует такое $q \in\{1, \ldots, r\}$ и такое $l \in\{0,1, \ldots, n-1\}$, что $a_{l}=0$ и $a_{l+i_{q}}=0$. Возможны четыре варианта:

$$
\begin{array}{ll}
l \equiv 0 \bmod k, & l+i_{q} \equiv 0 \bmod k, \\
l \equiv 0 \bmod k, & l+i_{q} \equiv j_{1} \bmod k, \\
l \equiv j_{1} \bmod k, & l+i_{q} \equiv 0 \bmod k, \\
l \equiv j_{1} \bmod k, & l+i_{q} \equiv j_{1} \bmod k .
\end{array}
$$

В каждом из указанных вариантов легко получить противоречие, пользуясь неравенствами $i_{q}<k$ и $j_{1}+i_{q}<k, q \in\{1, \ldots, r\}$ (напомним, что $j_{1} \neq k / 2$ ).

Те же рассуждения практически без изменения проводятся и для антимонотонных функций. Лемма доказана.

Теперь можно усилить предложение 2, получив однозначное представление произвольной циклически замкнутой биюнктивной функпии.

Предложение 4. Пусть $f$ - чиклически замкнутая биюнктивная функиия от $n$ переменных, отличная от константы и не имеющая импличент длины 1. Тогда возможен ровно один из трех вариантов:

- функчия $f$ мультиаффинна;

- функиия $f$ представляется в виде произведения $f=g h$, где

$$
g=\left(x_{0} \oplus x_{k} \oplus 1\right)^{*}, \quad k \mid n,
$$


причем $k$ однозначно определяется функчией $f u$

$$
h=\left(x_{0} \vee x_{i_{1}}\right)^{*} \ldots\left(x_{0} \vee x_{i_{r}}\right), \quad r>0, \quad 0<i_{1}<\ldots<i_{r} \leqslant k / 2,
$$

причем набор $\left\{i_{1}, \ldots, i_{r}\right\}$ однозначно определяется функиией $f$;

- функция $f$ представляется в виде произведения $f=g h$, где

$$
g=\left(x_{0} \oplus x_{k} \oplus 1\right)^{*}, \quad k \mid n,
$$

причем $k$ однозначно определяется функиией $f, u$

$$
h=\left(\bar{x}_{0} \vee \bar{x}_{i_{1}}\right)^{*} \ldots\left(\bar{x}_{0} \vee \bar{x}_{i_{r}}\right)^{*}, \quad r>0, \quad 0<i_{1}<\ldots<i_{r} \leqslant k / 2,
$$

причем набор $\left\{i_{1}, \ldots, i_{r}\right\}$ однозначно определяется функцией $f$.

Доказательство. Воспользуемся предложением 2 и представим $f$ в виде произведения функций $f=g h$, где $g$ - мультиаффинная, а $h$ - монотонная либо антимонотонная циклически замкнутые биюнктивные функции. Поскольку $f$ отлична от константы и не имеет имплицент длины 1, возможны два случая: $g=\left(x_{0} \oplus x_{i}\right)^{*}$, где число $2 i$ делит $n$, или $g=\left(x_{0} \oplus x_{i} \oplus 1\right)^{*}$, где число $i$ делит $n$.

Предположим, что $g=\left(x_{0} \oplus x_{i}\right)^{*}$. В силу того, что $g(0, \ldots, 0)=0$ и $g(1, \ldots, 1)=0$, не существует разложения $f=g^{\prime} h^{\prime}$ такого, что $g^{\prime}=\left(x_{0} \oplus x_{j} \oplus 1\right)^{*}$ и $h^{\prime}$ - монотонная или антимонотонная циклически замкнутая биюнктивная функщия. Остается заметить, что согласно лемме 4 и равенству (16) в случае, когда $h$ - монотонная или антимонотонная биюнктивная циклически замкнутая функция, функция $f$ является мультиаффинной биюнктивной циклически замкнутой функцией. Таким образом, для функции $f$ может быть выполнен только первый вариант.

Предположим теперь, что $g=\left(x_{0} \oplus x_{i} \oplus 1\right)^{*}$. Аналогично предыдущему рассуждению замечаем, что тогда не существует разложения для $f$ вида $f=g^{\prime} h^{\prime}$, где $g^{\prime}=\left(x_{0} \oplus x_{j}\right)^{*}$ и $h^{\prime}$ - монотонная или антимонотонная циклически замкнутая биюнктивная функция. Далее, если $h$ монотонна, то для $f$ выполняется второй вариант 2 в силу равенства (17). Если $h$ антимонотонна, то для $f$ выполняется третий вариант.

Однозначность определения $k$, делящего $n$, и набора $0<i_{1}<\ldots<i_{r} \leqslant k / 2$ следует из (18).

Осталось доказать, что для $f$ не могут выполняться одновременно варианты 1 и 2 (или варианты 1 и 3). Действительно, если для $f$ выполняются одновременно варианты 1 и 2 , то есть $f=\left(x_{0} \oplus x_{i} \oplus 1\right)^{*}$ и $f=h\left(x_{0} \oplus x_{j} \oplus 1\right)^{*}$, где $h$ монотонна, то $f(0, \ldots, 0)=1$ и $f(0, \ldots, 0)=0$ (поскольку $h(0, \ldots, 0)=0$ ); получаем противоречие.

Предложение доказано.

Доказательство теоремы 1. Число биюнктивных циклически замкнутых функций, равных константе или имеющих имплиценту длины 1 , равно 4. Число биюнктивных мультиаффинных циклически замкнутых функций от $n$ переменных, отличных от константы и не имеющих имплищент длины 1 , равно $\tau(n)$, когда $n$ нечетно, и $\tau(n)+\tau(n / 2)$, когда $n$ четно. Число функщий вида $f=g f$, где $g=\left(x_{0} \oplus x_{k} \oplus 1\right)^{*}, k \mid n$, и

$$
h=\left(x_{0} \vee x_{i_{1}}\right)^{*} \ldots\left(x_{0} \vee x_{i_{r}}\right)^{*}, \quad r>0, \quad 0<i_{1}<\ldots<i_{r} \leqslant k / 2,
$$

равно

$$
\sum_{k: k>0, k \mid n} 2^{\lfloor k / 2\rfloor-1}
$$


Число функций вида $f=g f$, где $g=\left(x_{0} \oplus x_{k} \oplus 1\right)^{*}, k \mid n$, и

$$
h=\left(\bar{x}_{0} \vee \bar{x}_{i_{1}}\right)^{*} \ldots\left(\bar{x}_{0} \vee \bar{x}_{i_{r}}\right)^{*}, \quad r>0, \quad 0<i_{1}<\ldots<i_{r} \leqslant k / 2,
$$

также равно (19). Таким образом,

$$
D(n)= \begin{cases}4-\tau(n)+\sum_{k: k>0, k \mid n} 2^{\lfloor(k+2) / 2\rfloor}, & \text { если } n \text { нечетно, } \\ 4-\tau(n)+\tau(n / 2)+\sum_{k: k>0, k \mid n} 2^{\lfloor(k+2) / 2\rfloor}, & \text { если } n \text { четно. }\end{cases}
$$

Теорема доказана.

В силу того, что число биюнктивных функций $b(n) \geqslant 2^{n^{2} / 2}$ (см. [4]), а число $D(n)$ есть $O\left(2^{n / 2}\right)$, очевидно, что доля циклически замкнутых функщий среди всех биюнктивных функций стремится к нулю с ростом числа $n$.

\section{3. Оценка числа биюнктивных функций, инвариантных относительно фиксированной подстановки}

В предыдущем параграфе получено точное значение числа биюнктивных функций, группы инерции которых содержат циклический сдвиг. Однако, если мы заменим подстановку сдвига на произвольную подстановку, то удается лишь оценить число таких функций.

Нам потребуется следующее свойство простых имплицент биюнктивной функщии.

Лемма 5. Пусть $f=\prod_{i=1}^{t} K_{i}$ - произвольная сокращенная КНФ и $s \in J(f)$. Тогда для любого $i \in\{1, \ldots, t\}$ существует $j \in\{1, \ldots, t\}$ такое, что $s\left(K_{i}\right)=K_{j}$.

Доказательство. Не ограничивая общности, проведем доказательство для случая $i=1$. Очевидно, что $s\left(K_{1}\right)$ является элементарной дизъюнкцией. Покажем, что $s\left(K_{1}\right)$ является имплицентой функции $f$. Поскольку $s \in J(f)$, справедливо равенство $f=s(f)$. Используя равенство

$$
s(f)=\prod_{i=1}^{t} s\left(K_{i}\right)
$$

получаем, что $s\left(K_{1}\right) f=s\left(K_{1}\right)\left(s\left(K_{1}\right) s\left(K_{2}\right) \ldots s\left(K_{t}\right)\right)=f$.

Теперь докажем, что $s\left(K_{1}\right)$ является простой имплицентой. Действительно, если существует имплицента $K^{\prime}$ такая, что $K^{\prime} s\left(K_{1}\right)=s\left(K_{1}\right)$ и $K^{\prime} f=f$, то для элементарной дизъюнкции $s^{-1}\left(K^{\prime}\right)$ верны равенства $s^{-1}\left(K^{\prime}\right) K_{1}=K_{1}$ и $s^{-1}\left(K^{\prime}\right) f=f$, и в силу простоты $K_{1}$ имплицента $s^{-1}\left(K^{\prime}\right)$ должна совпадать с $K_{1}$. Следовательно, $K^{\prime}=s\left(K_{1}\right)$, то есть $s\left(K_{1}\right)$ является простой имплицентой. Поскольку $K_{1}, \ldots, K_{t}$ - все простые имплиценты функции $f$, существует $j \in\{1, \ldots, t\}$ такое, что $s\left(K_{i}\right)=K_{j}$. Лемма доказана.

Обозначим $\operatorname{mob}(s)$ множество мобильных элементов подстановки $s \in S_{n}$, то есть множество таких $a \in\{0, \ldots, n-1\}$, что $s(a) \neq a$. Если $\operatorname{mob}(s) \subset I$, то без ограничения общности можно считать, что $s \in S(I)$. Пусть даны две подстановки $s_{1}$ и $s_{2}$ такие, что

$$
\operatorname{mob}\left(s_{1}\right) \subseteq I_{1}, \quad \operatorname{mob}\left(s_{2}\right) \subseteq I_{2}, \quad I_{1} \cap I_{2}=\varnothing, \quad I_{1} \cup I_{2}=\{0, \ldots, n-1\}
$$

Рассмотрим подстановку, равную упорядоченной паре подстановок $s_{1}$ и $s_{2}$, то есть $\left(s_{1}, s_{2}\right) \in S\left(I_{1} \times I_{2}\right)$, и циклическую группу $\left\langle\left(s_{1}, s_{2}\right)\right\rangle<S\left(I_{1} \times I_{2}\right)$, порожденную этой 
упорядоченной парой. Будем обозначать $\left[T\left(s_{1}, s_{2}\right)\right]$ некоторое множество орбит указанной группы подстановок (в том числе возможно и пустое множество).

Введем следующее обозначение. Если булева функция $g\left(x_{0}, \ldots, x_{n-1}\right)$ не зависит существенно от переменных с номерами, не входящими в некоторое множество $I \subset\{0,1, \ldots, n-1\}$, то запишем это таким образом:

$$
g\left(x_{0}, \ldots, x_{n-1}\right)=g\left(x_{i}, i \in I\right) .
$$

Будем говорить, что подстановка $s \in S_{n}$ представляется в виде произведения $k$ независимых подстановок $s_{1}, \ldots, s_{k}$, если $s=s_{1} \ldots s_{k}$ и $\operatorname{mob}\left(s_{i}\right) \cap \operatorname{mob}\left(s_{j}\right)=\varnothing$ при $i \neq j$, $i, j \in\{1, \ldots, k\}$.

Предложение 5. Пусть подстановка $s \in S_{n}$ представляется в виде произведения $k$ независимых подстановок $s_{1}, \ldots, s_{k}$. Пусть

$\operatorname{mob}\left(s_{i}\right) \subseteq I_{i}, \quad i=1, \ldots, k, \quad I_{i} \cap I_{j}=\varnothing, \quad i \neq j, \quad I_{1} \cup I_{2} \cup \ldots \cup I_{k}=\{0,1, \ldots, n-1\}$.

Тогда каждая биюнктивная функция $f$ от $n$ переменных, инвариантная относительно подстановки s, представляется в виде

$$
f\left(x_{0}, \ldots, x_{n-1}\right)=\prod_{e=1}^{k} f_{e}\left(x_{i}, i \in I_{e}\right) \prod_{1 \leqslant a<b \leqslant k} f_{a b}\left(x_{i}, i \in I_{a} \cup I_{b}\right),
$$

причем для любого $e=1, \ldots, k$ функция $f_{e}$ инвариантна относительно подстановки $s_{e}$ и для любых $a, b$ таких, что $1 \leqslant a<b \leqslant k$,

$$
f_{a b}\left(x_{i}, i \in I_{a} \cup I_{b}\right)=\prod_{T \in\left[T_{1}\left(s_{a}, s_{b}\right)\right]} \prod_{(i, j) \in T}\left(x_{i}^{\alpha_{T}} \vee x_{j}^{\beta_{T}}\right) \prod_{T \in\left[T_{2}\left(s_{a}, s_{b}\right)\right]} \prod_{(i, j) \in T}\left(x_{i} \oplus x_{j} \oplus \gamma_{T}\right),
$$

где $\left[T_{1}\left(s_{a}, s_{b}\right)\right],\left[T_{2}\left(s_{a}, s_{b}\right)\right]$ - непересекаючиеся семейства орбит группы подстановок $\left\langle\left(s_{a}, s_{b}\right)\right\rangle<S\left(I_{a} \times I_{b}\right)$.

Доказательство. Пусть $f$ - биюнктивная функция от $n$ переменных, причем $s \in J(f)$. Рассмотрим сокращенную КНФ функции $f$

$$
f=\prod_{i=1}^{t} K_{i}
$$

Поскольку $f$ - биюнктивная функщия, каждая из элементарных дизъюнкций $K_{i}$ существенно зависит от одного или двух переменных (см., например, [2]). Разобьем множество всех $K_{i}$ на $k+\left(\begin{array}{c}k \\ 2\end{array}\right)$ непересекающихся групп $U_{1}, \ldots, U_{k}, U_{12}, U_{13}, \ldots, U_{k-1 k}$ следующим образом: в группу $U_{j}$ входят только элементарные дизъюнкции от переменных с номерами из множества $I_{j}, j \in 1, \ldots, k$; в группу $U_{i j}$ - дизъюнкции длины 2 , в которых одна переменная имеет номер из $I_{i}$, а вторая переменная номер из $I_{j}$. Таким образом, функщия $f$ представляется в виде произведения

$$
\begin{aligned}
f\left(x_{0}, \ldots, x_{n-1}\right) & =\left(\prod_{e=1}^{k} \prod_{K \in U_{e}} K\right)\left(\prod_{1 \leqslant a<b \leqslant k} \prod_{K \in U_{a b}} K\right) \\
& =\prod_{e=1}^{k} f_{e}\left(x_{i}, i \in I_{c}\right) \prod_{1 \leqslant a<b \leqslant k} f_{a b}\left(x_{i}, i \in I_{a} \cup I_{b}\right) .
\end{aligned}
$$


Поскольку каждая из функций $f_{e}, f_{a b}$ задается 2-КНФ, то все они являются биюнктивными функциями. Кроме того, из леммы 5 следует, что для каждой из функций $f_{e}$ справедливо включение $s_{e} \in J\left(f_{e}\right)$.

Остается изучить функции $f_{a b}$. Опять воспользуемся леммой 5 и получим, что если дизъюнкция $\left(x_{\mu}^{\alpha} \vee x_{v}^{\beta}\right)$ лежит во множестве $U_{a b}$, то в этом множестве лежат все дизъюнкции вида

$$
\left(x_{s_{a}}(\mu)^{\alpha} \vee x_{s_{b}(\nu)}^{\beta}\right),\left(x_{s_{a}^{2}(\mu)}^{\alpha} \vee x_{s_{b}^{2}(\nu)}^{\beta}\right), \ldots,
$$

то есть все такие дизъюнкции $\left(x_{\omega}^{\alpha} \vee x_{\sigma}^{\beta}\right)$, что $(\omega, \sigma)$ принадлежат орбите элемента $(\mu, \nu)$ в группе $\left\langle\left(s_{a}, s_{b}\right)\right\rangle<S\left(I_{a} \times I_{b}\right)$. Таким образом, функция $f_{a b}$ имеет вид

$$
\begin{aligned}
f_{a b}\left(x_{i}, i \in I_{a} \cup I_{b}\right)= & \prod_{T \in\left[O_{1}\left(s_{a}, s_{b}\right)\right]} \prod_{(i, j) \in T}\left(x_{i}^{\vee} x_{j}\right) \prod_{T \in\left[O_{2}\left(s_{a}, s_{b}\right)\right]} \prod_{(i, j) \in T}\left(\bar{x}_{i} \vee x_{j}\right) \\
\times & \prod_{T \in\left[O_{3}\left(s_{a}, s_{b}\right)\right]} \prod_{(i, j) \in T}\left(x_{i}^{\vee} \bar{x}_{j}\right) \prod_{T \in\left[O_{4}\left(s_{a}, s_{b}\right)\right]} \prod_{(i, j) \in T}\left(\bar{x}_{i} \vee \bar{x}_{j}\right),
\end{aligned}
$$

где семейства орбит $\left[O_{1}\left(s_{a}, s_{b}\right)\right],\left[O_{2}\left(s_{a}, s_{b}\right)\right],\left[O_{3}\left(s_{a}, s_{b}\right)\right],\left[O_{4}\left(s_{a}, s_{b}\right)\right]$ могут пересекаться. Однако отметим, что некоторые пересечения обязаны быть пустыми:

$$
\begin{aligned}
{\left[O_{1}\left(s_{a}, s_{b}\right)\right] \cap\left[O_{2}\left(s_{a}, s_{b}\right)\right] } & =\left[O_{1}\left(s_{a}, s_{b}\right)\right] \cap\left[O_{3}\left(s_{a}, s_{b}\right)\right] \\
& =\left[O_{2}\left(s_{a}, s_{b}\right)\right] \cap\left[O_{4}\left(s_{a}, s_{b}\right)\right] \\
& =\left[O_{3}\left(s_{a}, s_{b}\right)\right] \cap\left[O_{4}\left(s_{a}, s_{b}\right)\right]=\varnothing,
\end{aligned}
$$

так как у функция $f$ не могут быть простыми имплицентами одновременно дизъюнкции $\left(x_{i}^{\alpha} \vee x_{j}^{\beta}\right)$ и $\left(x_{i}^{\alpha} \vee x_{j}^{\beta \oplus 1}\right)$, поскольку их произведение равно $x_{i}^{\alpha}$, а следовательно, $f x_{i}^{\alpha}=f$, то есть имплиценты $\left(x_{i}^{\alpha} \vee x_{j}^{\beta}\right)$ и $\left(x_{i}^{\alpha} \vee x_{j}^{\beta \oplus 1}\right)$ не простые.

Определим семейства орбит $\left[t_{1}\left(s_{a}, s_{b}\right)\right]$ и $\left[t_{2}\left(s_{a}, s_{b}\right)\right]$, полагая

$$
\begin{aligned}
& {\left[T_{1}\left(s_{a}, s_{b}\right)\right]=\bigcup_{l=1}^{4}\left(\left[O_{l}\left(s_{a}, s_{b}\right)\right]\right) \backslash\left(\left(\left[O_{1}\left(s_{a}, s_{b}\right)\right] \cap\left[O_{4}\left(s_{a}, s_{b}\right)\right] \cup\left[O_{2}\left(s_{a}, s_{b}\right)\right] \cap\left[O_{3}\left(s_{a}, s_{b}\right)\right]\right)\right),} \\
& {\left[T_{2}\left(s_{a}, s_{b}\right)\right]=\left(\left(\left[O_{2}\left(s_{a}, s_{b}\right)\right] \cap\left[O_{4}\left(s_{a}, s_{b}\right)\right] \cup\left[O_{3}\left(s_{a}, s_{b}\right)\right] \cap\left[O_{4}\left(s_{a}, s_{b}\right)\right]\right) .\right.}
\end{aligned}
$$

Семейства $\left[T_{1}\left(s_{a}, s_{b}\right)\right]$ и $\left[T_{2}\left(s_{a}, s_{b}\right)\right]$ не пересекаются. Воспользовавшись равенством

$$
\left(x_{i}^{\alpha} \vee x_{j}^{\beta}\right)\left(x_{i}^{\alpha \oplus 1} \vee x_{j}^{\beta \oplus 1}\right)=\left(x_{i} \oplus x_{j} \oplus \alpha \oplus \beta\right),
$$

получаем, что

$$
\prod_{T \in\left[T_{2}\left(s_{a}, s_{b}\right)\right]} \prod_{(i, j) \in T}\left(x_{i}^{\alpha_{T}} \vee x_{j}^{\beta_{T}}\right)\left(x_{i}^{\alpha \oplus 1} \vee x_{j}^{\beta \oplus 1}\right)=\prod_{T \in\left[T_{2}\left(s_{a}, s_{b}\right)\right]} \prod_{(i, j) \in T}\left(x_{i} \oplus x_{j} \oplus \gamma_{T}\right) .
$$

Таким образом, функция $f_{a b}$ записана в требуем виде. Утверждение доказано.

Напомним, что в начале статьи мы обозначили через $D(s, n)$ число биюнктивных функций от $n$ переменных, содержащих в своей группе инерции подстановку $s$. Кроме этого, пусть $d_{a b}$ - число различных орбит группы $\left\langle\left(s_{a}, s_{b}\right)\right\rangle<S\left(I_{a} \times I_{b}\right)$. Справедливо следующее утверждение. 
Следствие 2. При условиях предложения 5 справедлива оченка

$$
D(s, n) \leqslant 7^{\sum_{1 \leqslant a<b \leqslant k} d_{a b}} \prod_{i=1}^{k} D\left(s_{i},\left|I_{i}\right|\right) .
$$

Доказательство. Каждая из функщий $f_{e}$ может принимать не более $D\left(s_{e},\left|I_{e}\right|\right)$ значений, так как $s_{e} \in J\left(f_{e}\right), e=1, \ldots, k$. Каждая из функций $f_{a b}$ может принимать не более $7^{d_{a b}}$ значений, так как из всех $d_{a b}$ орбит группы $\left\langle\left(s_{a}, s_{b}\right)\right\rangle<S\left(I_{a} \times I_{b}\right)$ необходимо выбрать 7 подмножеств (возможно пустых):

- орбиты, которых нет в семействах $\left[T_{1}\left(s_{a}, s_{b}\right)\right],\left[T_{2}\left(s_{a}, s_{b}\right)\right]$;

- орбиты, для которых в функщию $f_{a b}$ входит дизъюнкция $\left(x_{i} \vee x_{j}\right)$;

- орбиты, для которых в функцию $f_{a b}$ входит дизъюнкщия $\left(\bar{x}_{i} \vee x_{j}\right)$;

- орбиты, для которых в функцию $f_{a b}$ входит дизъюнкция $\left(x_{i} \vee \bar{x}_{j}\right)$;

- орбиты, для которых в функцию $f_{a b}$ входит дизъюнкщия $\left(\bar{x}_{i} \vee \bar{x}_{j}\right)$;

- орбиты, для которых в функцию $f_{a b}$ входит сумма $\left(x_{i} \oplus x_{j}\right)$;

- орбиты, для которых в функцию $f_{a b}$ входит сумма $\left(x_{i} \oplus x_{j} \oplus 1\right)$.

Разбить множество из $d_{a b}$ элементов на 7 непересекающихся подмножеств можно ровно $7^{d_{a b}}$ способами. Следствие доказано.

Найти величины $d_{a b}$ можно по лемме Бернсайда. Однако в некоторых случаях можно сразу выписать итоговое значение числа орбит. Число циклически замкнутых биюнктивных функций от $n$ переменных в предыдущих параграфах мы обозначали $C(n)$. Предположим, что $s=s_{1} \ldots s_{k}$ - разложение подстановки $s \in S_{n}$ на независимые циклы $s_{i} \in S\left(I_{i}\right)$, $i=1, \ldots, k$. Тогда $D\left(s_{i},\left|I_{i}\right|\right)=C\left(\left|I_{i}\right|\right)$, и в каждой группе мощности всех орбит совпадают и равны наименьшему общему кратному чисел $\left|I_{a}\right|$ и $\left|I_{b}\right|$. Тогда очевидно, что $d_{a b}=\left(\left|I_{a}\right|,\left|I_{b}\right|\right)$, откуда следует утверждение теоремы 2 . Повторим ее формулировку.

Пусть подстановка $s$ разлагается на $k$ независимых циклов длины $l_{1}, \ldots, l_{k}$. Тогда

$$
D(s, n) \leqslant 7^{\sum_{1 \leqslant a<b \leqslant k}\left(l_{a}, l_{b}\right)} \prod_{i=1}^{k} D\left(l_{i}\right) .
$$

Сделаем несколько замечаний относительно полученной оценки (20). Иногда эта оценка является точным значением, например, в случае, когда $s$ - подстановка сдвига. Однако, если взять в качестве $s$ тождественную подстановку (и таким образом оценить число всех биюнктивных функций от $n$ переменных), то оценка будет составлять $\prod_{i=1}^{n} 4 \cdot 7\left(\begin{array}{l}n \\ 2\end{array}\right)$, что, очевидно, больше известной оценки $2^{n^{2}}$ (см., например, [5]). 


\section{Список литературы}

1. Schaefer T., Complexity of satisfiability problems. In: Proc. 10 Annual ACM Symp. on Theory of Computing, 1978, pp.,216-226.

2. Данцин Е. Я., Алгоритмика задачи выполнимости. Bопросы киберн. (1987) 131, 7-21.

3. Гизунов С. А., Носов В. А., О классификации всех булевых функций 4-х переменных по классам Шеффера. Обозрение прикладной и промышленной математики (1995) 2, №3, 440-467.

4. Горшков С. П., О сложности задачи нахождения числа решений систем булевых уравнений. Дискретная математика (1996) 8, №1, 72-85.

5. Горшков С. П., Применение теории $N P$-полных задач для оценки сложности решения булевых уравнений. Обозрение прикладной и промышиенной математики (1995) 2, №3, 325-398.

6. Яблонский С. В., Введение в дискретную математику. Наука, Москва, 1997.

7. Тарасов А. В., О свойствах функций, представимых в виде 2-КНФ. Дискретная математика (2001) 13, №4, 99-115.

Статья поступила 25.04.2002. 